\title{
Reconstruction of an Unknown Boundary Portion from Cauchy Data in $N$-dimensions
}

\author{
October 26, 2004 \\ Kurt Bryan, Rose-Hulman Institute of Technology \\ Lester Caudill, University of Richmond
}

\begin{abstract}
We consider the inverse problem of determining the shape of some inaccessible portion of the boundary of a region in $n$ dimensions from Cauchy data for the heat equation on an accessible portion of the boundary. The inverse problem is quite ill-posed, and nonlinear. We develop a Newton-like algorithm for solving the problem, with a simple and efficient means for computing the required derivatives, develop methods for regularizing the process, and provide computational examples.
\end{abstract}

\section{Introduction}

Let $\Omega$ be a bounded and simply connected region in $\mathbb{R}^{n}$, with boundary $\partial \Omega$ consisting of two surfaces $\Gamma$ and $S_{0}$, and let $u=u(t, x)$ (the temperature of $\Omega$ ) satisfy the initial-boundary value problem

$$
\begin{aligned}
\frac{\partial u}{\partial t}-\triangle_{x} u & =0, \quad x \in \Omega, 0<t<T ; \\
\frac{\partial u}{\partial \eta} & =g(t, x), \quad x \in \Gamma, 0<t<T ; \\
\frac{\partial u}{\partial \eta} & =0, \quad x \in S_{0}, 0<t<T ; \\
u(0, x) & =0, \quad x \in \Omega
\end{aligned}
$$

where $\eta$ denotes a unit outward normal vector on $\partial \Omega$. The function $g$ represents an input heat flux (at least after suitable rescaling) on $\Gamma$, while we assume that the surface $S_{0}$ is perfectly insulating. Given suitable $\Gamma, S_{0}$, and $g$, determining $u$ on $\Omega$ over a prescribed time interval is a well-posed problem.

We consider the inverse problem in which the surface $\Gamma$ is accessible for temperature/flux measurements, while $S_{0}$ is considered inaccessible, and is to be determined from the Cauchy data $g(t, x)$ and

$$
u(t, x) \text { for } x \in \Gamma \text { and } t \in(0, T) \text {. }
$$


We often refer to $\Gamma$ as the "front surface" of $\Omega$ and $S_{0}$ as the "back surface." In this paper, we present a new algorithm for estimating $S_{0}$ from given Cauchy data. This algorithm, based on a Newton-type method, offers a number of advantages:

- It is applicable to a quite general class of domains.

- As derived, it can be applied to domains in $\mathbb{R}^{n}$, for any $n \geq 2$.

When investigating an inverse problem, the three issues of uniqueness, stability, and reconstruction are paramount. Uniqueness for the present inverse problem, for any notidentically-zero $g$ has been established previously (see [5]). Theoretical estimates on the stability of $S_{0}$ with respect to the Cauchy data on $\Gamma$ presently exist in the cases where $n=2$ or $n=3$. The first of these to apply to $\mathbb{R}^{3}$ was proved by Vessella in [11], which addresses the case where the roles of Neumann and Dirichlet data on $\Gamma$ are reversed. In this setting, a logarithmic stability estimate is established, under the assumption that the Dirichlet data prescribed on $(0, T] \times \partial \Omega$ is monotone with respect to time. More recently, logarithmic stability was shown for the inverse problem (1)-(5) by Canuto, Rosset, and Vessella [7].

Reconstruction methods for boundary identification problems for the heat equation have been proposed and numerically tested by Banks, Kojima, and Winfree $[1,2]$ in the case where the domain $\Omega$ is a rectangle in $\mathbb{R}^{2}$, by Chapko, Kress, and Yoon [8] in the case where $\Omega$ is the unit disk in $\mathbb{R}^{2}$, and by Bryan and Caudill $[4,3]$ in the case where $\Omega$ is a strip in $\mathbb{R}^{2}$. To our knowledge, no algorithm applicable in $\mathbb{R}^{n}$ has yet been proposed and tested.

The present paper is organized as follows: In section 2, we introduce notation and assumptions. In section 3, we establish an important differentiability result. Section 4 is devoted to establishing a numerical algorithm, including derivation of an explicit form for the Jacobian of a featured functional. In section 5, we present some numerical experiments demonstrating the effectiveness of the algorithm.

\section{Preliminaries}

\section{Notation}

For a domain $\Omega \subseteq \mathbb{R}^{n}$ or surface $S \subseteq \mathbb{R}^{n-1}$, we will use the notation $\Omega_{T}$ to denote $(0, T) \times \Omega$, $S_{T}$ to denote $(0, T) \times S$, etc. We will require the following function spaces:

- $C_{B}(\bar{\Omega})$ : The space of bounded and continuous functions on $\bar{\Omega}$, equipped with the supremum norm.

- $L_{2}\left(\Omega_{T}\right)$ : The space of square-integrable functions on $\Omega_{T}$, with norm $\|u\|_{2, \Omega_{T}}=\left(\int_{0}^{T} \int_{\Omega}|u|^{2} d x d t\right)^{\frac{1}{2}}$. 
- $W_{2}^{2,1}\left(\Omega_{T}\right)$ : The Sobolev space of functions on $\Omega_{T}$ that have square-integrable derivatives through order two in space and order one in time, with norm

$$
\|u\|_{2, \Omega_{T}}^{(2)}=\|u\|_{2, \Omega_{T}}+\left\|u_{t}\right\|_{2, \Omega_{T}}+\sum_{j=1}^{n}\left\|u_{x_{j}}\right\|_{2, \Omega_{T}}+\sum_{k=1}^{n} \sum_{j=1}^{n}\left\|u_{x_{j} x_{k}}\right\|_{2, \Omega_{T}} .
$$

Additionally, we will utilize the trace space $W_{2}^{\frac{1}{2}, \frac{1}{4}}\left(\partial \Omega_{T}\right)$, with its norm $\|u\|_{2, \partial \Omega_{T}}^{\left(\frac{1}{2}\right)}$. These are properly defined in terms of a covering of $\partial \Omega_{T}$, and will not be given in detail here. (For a precise definition, see [10], page 81.) For our purposes, we will think of this space as the trace space on $\partial \Omega_{T}$ of $u_{x_{j}}$, for $u \in W_{2}^{2,1}\left(\Omega_{T}\right)$.

Finally, given a domain $D \subseteq \mathbb{R}^{n}$, define the function class $O^{2}(D)$ as follows:

$$
O^{2}(D) \equiv\left\{f \in C^{1}(D, \mathbb{R}): f^{\prime \prime} \text { exists as a bounded function on } D\right\} .
$$

One note on domains: We say that the boundary $\partial D$ of a domain $D$ is of class $O^{2}$ if each point in $\partial D$ possesses an open neighborhood $\omega \subseteq \partial D$ which can be represented in locally as the graph of a function in the class $O^{2}(B)$ for some set $B \subset \mathbb{R}^{n-1}$.

\section{Assumptions}

We will assume that the spatial domain $\Omega$, for a suitable choice of coordinate system, can be constructed in the following way: Let $\hat{\Omega}$ be a bounded and simply connected region in $\mathbb{R}^{n-1}$, and let $x_{n}=\gamma(\hat{x})$ be a real-valued function that belongs to $O^{2}(\overline{\hat{\Omega}})$, where $\hat{x}=$ $\left(x_{1}, x_{2}, \ldots, x_{n-1}\right)$. We use $\Gamma$ (the front-surface) to denote the graph of $\gamma$. We define the set $\mathcal{P}_{\gamma}$ of admissible back surfaces as follows: $\mathcal{P}_{\gamma}$ is the set of all surfaces $S$ which can be represented as the graph $x_{n}=\sigma(\hat{x})$ of a function $\sigma$ which satisfies the following properties:

- $\sigma=\gamma$ on $\partial \overline{\hat{\Omega}}$.

- $\sigma(\hat{x})<\gamma(\hat{x})$ for all $\hat{x}$ in the interior of $\hat{\Omega}$.

- The boundary of the domain $\Omega$ enclosed by the graphs of $\gamma$ and $\sigma$ is of class $O^{2}$.

For any $\sigma \in \mathcal{P}_{\gamma}$ the surface $S$ defined by $x_{n}=\sigma(\hat{x})$ over $\hat{\Omega}$ and the surface $\Gamma$ together enclose a bounded and simply connected domain $\Omega$ in $\mathbb{R}^{n}$.

Assume that the Dirichlet data $u(t, x)$ for the unknown back surface $S_{0}$ is given for $x \in \Gamma, 0<t<T$. We denote this "measured" data by $d(t, x)$. For a given arbitrary $S \in \mathcal{P}_{\gamma}$, we denote by $u_{S}$ the solution to the IBVP (1)-(4) (with $S_{0}=S$ ).

\footnotetext{
${ }^{1}$ The results of this paper actually extend to include a class of unbounded domains $\Omega$ as well. See Section 6 for further discussion.
} 


\section{Domain Differentiability}

We now establish the differentiability (in the sense of Gateaux) of the solution $u_{S}$ of (1)-(4) with respect to $S$ at $S_{0}$ in the direction $\alpha \in \mathcal{A}$, where $\mathcal{A}$, the set of admissible directions, is the subset of $O^{2}(\hat{\Omega})$ consisting of functions which are zero, along with their first partial derivatives, on $\hat{\partial}$. In so doing, we will require the following solvability result, Theorem 3.1 below, for parabolic IBVPs of the form

$$
\begin{aligned}
a(x) \frac{\partial u}{\partial t} & -\nabla \cdot(\mathbf{A}(x) \nabla u)=f, \quad \text { on } \Omega_{T} ; \\
u(0, x) & =0, \quad \text { on } \bar{\Omega}, \\
\frac{\partial u}{\partial \nu} & =h(t, x), \quad \text { on } \partial \Omega_{T} .
\end{aligned}
$$

For a proof of this result, see [10] (Theorem IV.9.1, pages 341 and 351).

Theorem 3.1 Let the following hold:

- $\Omega \subseteq \mathbb{R}^{n}$ has boundary of class $O^{2}$.

- $a(x) \in C_{B}(\bar{\Omega})$ with $a(x) \geq \delta>0 \forall x \in \bar{\Omega}$, for some $\delta>0$.

- Each entry of the matrix-valued function $\mathbf{A}(x)$ is an element of $C_{B}(\bar{\Omega})$, and there exist positive constants $\alpha$ and $\beta$ so that $\mathbf{A}(x)$ satisfies the uniform ellipticity condition

$$
\alpha|\xi|^{2} \leq \xi^{T} \mathbf{A}(x) \xi \leq \beta|\xi|^{2}
$$

for each $\xi \in \mathbb{R}^{n}$.

- $f \in L_{2}\left(\Omega_{T}\right)$.

- $h \in W_{2}^{\frac{1}{2}, \frac{1}{4}}\left(\partial \Omega_{T}\right)$ with $h(0, x)=0$ on $\partial \Omega$.

Then the IBVP (6)-(8) has a unique solution $u \in W_{2}^{2,1}\left(\Omega_{T}\right)$. Moreover, there is a constant $c>0$ (independent of $u$ ) such that

$$
\|u\|_{2, \Omega_{T}}^{(2)} \leq c\left(\|f\|_{2, \Omega_{T}}+\|h\|_{2, \partial \Omega_{T}}^{\left(\frac{1}{2}\right)}\right) .
$$

Let $\gamma$ be such that $\mathcal{P}_{\gamma}$ is non-empty and let $\sigma \in \mathcal{P}_{\gamma}$, thus forming the domain $\Omega$ with $\partial \Omega=\Gamma \cup S_{0}$. We begin by considering a perturbation of $\sigma$ by $\epsilon \alpha$ for some $\alpha \in \mathcal{A}$ with $\epsilon>0$ sufficiently small so that $\sigma+\epsilon \alpha \in \mathcal{P}_{\gamma}$. (It follows from the definitions of $\mathcal{P}_{\gamma}$ and $\mathcal{A}$ 
that, given $\alpha \in \mathcal{A}$, there exists $\epsilon_{0}>0$ such that $\epsilon \in\left[0, \epsilon_{0}\right]$ implies $\sigma+\epsilon \alpha \in \mathcal{P}_{\gamma}$.) Under this perturbation, the IBVP (1)-(4) becomes

$$
\begin{aligned}
\frac{\partial u_{\epsilon}}{\partial t}-\triangle_{x} u_{\epsilon} & =0, \quad x \in \Omega_{\epsilon}, 0<t<T ; \\
\frac{\partial u_{\epsilon}}{\partial \eta_{x}} & =g(t, x), \quad x \in \Gamma, 0<t<T ; \\
\frac{\partial u_{\epsilon}}{\partial \eta_{x}} & =0, \quad x \in S_{0}+\epsilon S, 0<t<T ; \\
u_{\epsilon}(0, x) & =0, \quad x \in \Omega_{\epsilon},
\end{aligned}
$$

where $S_{0}+\epsilon S$ denotes the graph of $\sigma+\epsilon \alpha$, and $\Omega_{\epsilon}$ denotes the region bounded by $\Gamma$ and $S_{0}+\epsilon S$.

It will be advantageous to have both (1)-(4) and (10)-(13) formulated on the same domain $\Omega$. To this end, we introduce the spatial change-of-variables $\Phi=\Phi(x, \epsilon)=\Phi\left(\hat{x}, x_{n}, \epsilon\right)$ defined by

$$
\left(y_{1}, \ldots, y_{n}\right)=\Phi\left(x_{1}, \ldots, x_{n} ; \epsilon\right)=\left(\phi_{1}(x, \epsilon), \ldots, \phi_{n}(x, \epsilon)\right)
$$

where

$$
\begin{aligned}
\phi_{j}(x, \epsilon) & =x_{j}, \quad 1 \leq j \leq n-1, \\
\phi_{n}(x, \epsilon) & =x_{n}+\left(\frac{\epsilon \alpha(\hat{x})}{\gamma(\hat{x})-\sigma(\hat{x})-\epsilon \alpha(\hat{x})}\right)\left(x_{n}-\gamma(\hat{x})\right) .
\end{aligned}
$$

We denote the Jacobian matrix of $\Phi$ by $\Psi$. It is easy to check that

$$
\Phi(\hat{x}, \gamma(\hat{x}), \epsilon)=(\hat{x}, \gamma(\hat{x}))
$$

and

$$
\Phi(\hat{x}, \sigma(\hat{x})+\epsilon \alpha(\hat{x}), \epsilon)=(\hat{x}, \sigma(\hat{x})),
$$

i.e. $\Phi(x, \epsilon)$ leaves $\Gamma$ fixed, and maps $S_{0}+\epsilon S$ to $S_{0}$. Consequently, $\Phi(x, \epsilon)$ maps $\Omega_{\epsilon}$ to $\Omega$.

Applying this change-of-variables to the IBVP (10)-(13) results in an IBVP for $v \equiv$ $u_{\epsilon} \circ \Phi^{-1}$ :

$$
\begin{aligned}
\rho(y, \epsilon) \frac{\partial v}{\partial t} & -\nabla_{y} \cdot\left(\kappa(y, \epsilon) \nabla_{y} v\right)=0, \quad y \in \Omega, 0<t<T ; \\
\frac{\partial v}{\partial \nu_{y}} & =g(t, y), \quad y \in \Gamma, 0<t<T ; \\
\frac{\partial v}{\partial \nu_{y}} & =0, \quad y \in S_{0}, 0<t<T ; \\
v(0, y) & =0, \quad y \in \Omega,
\end{aligned}
$$


where

$$
\begin{aligned}
\rho(y, \epsilon) & =\frac{1}{\operatorname{det}(\Psi(y, \epsilon))}, \\
\kappa(y, \epsilon) & =\rho(y, \epsilon) \Psi(y, \epsilon)(\Psi(y, \epsilon))^{T}, \\
\nu_{y} & =\nu_{y}(y, \epsilon)=\Psi(y, \epsilon) \eta_{x}\left(\Phi^{-1}(y, \epsilon)\right) .
\end{aligned}
$$

We summarize some useful properties of these functions in the next result, whose proof is a direct consequence of the definition (14) of $\Phi$.

Lemma 3.1 There exists an $\epsilon_{1}>0$ for which the following properties hold for all $0<\epsilon<$ $\epsilon_{1}$ :

- $\rho, \nu$, and each entry of the matrix $\kappa$ are elements of $C_{B}(\Omega)$.

- Each entry of the vector $\nabla \cdot \kappa$ exists as a bounded function on $\Omega$. (This is a consequence of the membership of $\gamma, \sigma$, and $\alpha$ in the class $O^{2}(\hat{\Omega})$.)

- As $\epsilon \rightarrow 0$, each of the following quantities goes to zero:

$-\|1-\rho\|_{\infty}$,

- $\|\kappa-I\|_{\infty}$ (The maximum of the supremum norms of the entries of the matrix $\kappa-I$.),

- $\|\nabla \cdot(\kappa-I)\|_{\infty}$ (The maximum of the supremum norms of the entries of the vector $\nabla \cdot(\kappa-I)$.).

Moreover, $\rho, \kappa$, and $\nu_{y}$ are each Gateaux differentiable with respect to $S$, and the resulting derivatives are bounded and continuous on $\hat{\Omega}$.

The main result of this section may now be stated.

Theorem 3.2 Under the conditions specified, the solution u of (1)-(4) is Gateaux differentiable with respect to $S$, and its derivative $w$ at $S_{0}$ in the direction of $\alpha$ is the unique solution of the IBVP

$$
\begin{aligned}
\frac{\partial w}{\partial t} & -\Delta w=\nabla \cdot\left(\kappa_{\epsilon}(x, 0) \nabla u\right)-\rho_{\epsilon}(x, 0) \frac{\partial u}{\partial t}, \quad \text { on } \Omega_{T} \\
\frac{\partial w}{\partial \eta} & =-\frac{\partial u}{\partial \nu_{\epsilon}}, \quad \text { on } \Gamma_{T} ; \\
\frac{\partial w}{\partial \eta} & =-\frac{\partial u}{\partial \nu_{\epsilon}}, \quad \text { on } S_{T} ; \\
w(0, x) & =0, \quad \text { on } \bar{\Omega} ;
\end{aligned}
$$


where $\rho_{\epsilon}, \kappa_{\epsilon}$, and $\nu_{\epsilon}$ are the Gateaux derivatives (in the direction of $\alpha$ ) of $\rho, \kappa$, and $\nu_{y}$, respectively. Here u represents the solution to (1)-(4) on $\Omega$.

Proof. From the assumptions, it is clear from Theorem 3.1 that (22)-(25) has a unique solution $w \in W_{2}^{2,1}\left(\Omega_{T}\right)$. The present proof consists in showing that

$$
\left\|\frac{u-v}{\epsilon}-w\right\|_{2, \Omega_{T}}^{(2)} \rightarrow 0 \text { as } \epsilon \rightarrow 0
$$

where $v$ satisfies (15)-(18).

Set $z \equiv u-v$. Then, $z$ is the unique solution in $W_{2}^{2,1}\left(\Omega_{T}\right)$ of the IBVP

$$
\begin{aligned}
\frac{\partial z}{\partial t} & -\Delta z=\nabla \cdot(\kappa-I) \nabla v-(\rho-1) \frac{\partial v}{\partial t}, \quad \text { on } \Omega_{T} ; \\
\frac{\partial z}{\partial \eta} & =g(t, y)-g(t, x)+\nabla v \cdot(\eta-\nu), \quad \text { on } \Gamma_{T} ; \\
\frac{\partial z}{\partial \eta} & =\nabla v \cdot(\eta-\nu), \quad \text { on } S_{T} ; \\
z(0, x) & =0, \text { on } \bar{\Omega} .
\end{aligned}
$$

By applying (9) to this IBVP, and incorporating the results of Lemma 3.1 and the fact that $v \in W_{2}^{2,1}\left(\Omega_{T}\right)$, we conclude that

$$
\|z\|_{2, \Omega_{T}}^{(2)} \rightarrow 0 \text { as } \epsilon \rightarrow 0
$$

Now, set $q \equiv \frac{z}{\epsilon}-w$ with $\epsilon>0$, so that $q \in W_{2}^{2,1}\left(\Omega_{T}\right)$ is the unique solution of the IBVP

$$
\begin{aligned}
\frac{\partial q}{\partial t} & -\Delta q=\nabla \cdot\left(\frac{1}{\epsilon}(\kappa-I)-\kappa_{\epsilon}\right) \nabla v+\left(\rho_{\epsilon}-\frac{1}{\epsilon}(\rho-1)\right) \frac{\partial v}{\partial t}+\nabla \cdot\left(\kappa_{\epsilon} \nabla z\right)+\rho_{\epsilon} \frac{\partial z}{\partial t} \\
\frac{\partial q}{\partial \eta} & =\frac{1}{\epsilon}(g(t, y)-g(t, x))+\nabla v \cdot\left(\nu_{\epsilon}-\frac{1}{\epsilon}(\eta-\nu)\right)-\frac{\partial z}{\partial \nu_{\epsilon}}, \quad \text { on } \Gamma_{T} ; \\
\frac{\partial q}{\partial \eta} & =\nabla v \cdot\left(\nu_{\epsilon}-\frac{1}{\epsilon}(\eta-\nu)\right)-\frac{\partial z}{\partial \nu_{\epsilon}}, \quad \text { on } S_{T} ; \\
q(0, x) & =0, \quad \text { on } \bar{\Omega} .
\end{aligned}
$$

By virtue of (26), the existence of $\rho_{\epsilon}, \kappa_{\epsilon}$, and $\nu_{\epsilon}$, and the finiteness of $\|v\|_{2, \Omega_{T}}^{(2)}$, the bound (9), applied to the IBVP (27)-(30), shows that $\|q\|_{2, \Omega_{T}}^{(2)} \rightarrow 0$ as $\epsilon \rightarrow 0$, which completes the proof. 


\section{Reconstruction Algorithm}

Given Dirichlet data $d(t, x)$ on $\Gamma_{T}$, we seek an admissible back surface $S$ for which $u_{S}(t, x)=$ $d(t, x)$ on $\Gamma_{T}$. Recognizing the inherent ill-posedness of this inverse problem, we relax this requirement as follows: We select a linearly independent set of functions $\left\{\rho_{j}(t, x)\right\}_{j=1}^{J}$ defined on $\Gamma_{T}$, and seek an $\alpha \in \mathcal{P}_{\gamma}$ for which

$$
\int_{\Gamma_{T}}\left(u_{S}-d\right) \rho_{j} d s d t=0, \forall j
$$

that is, we seek only to match certain select moments of the data $d$ and function $u_{S}$. This approach has been used before, e.g., in [4] and [9], especially to study and regularize the ill-posedness of certain inverse problems.

We thus define a vector-valued functional $F: \mathcal{P}_{\gamma} \rightarrow \mathbb{R}^{J}$ as $F(S)=\left\{F_{j}(S)\right\}_{j=1}^{J}$, where, for each $j$,

$$
F_{j}(S)=\int_{\Gamma_{T}}\left(u_{S}-d\right) \rho_{j} d s d t
$$

We present an explicit representation for the Jacobian of this operator, which will permit implementation of a Newton-type method for finding a root of $F$ in $\mathcal{P}_{\gamma}$. Note also that by taking the $\rho_{j}$ to be delta-functions this approach can be used to implement a straightforward point-by-point matching of data $d(t, x)$ and tentative solution $u_{S}(t, x)$, if desired.

A direct calculation yields the following:

Theorem 4.1 The Jacobian $F_{j}^{\prime}\left(S_{0}\right) \alpha$, of the operator $F_{j}$ defined in (31) has the form

$$
F_{j}^{\prime}\left(S_{0}\right) \alpha=\int_{\Gamma_{T}} w \rho_{j} d s d t
$$

where $w$ is the Gateaux derivative of $u_{S}$ at $S_{0}$ in the direction $\alpha$.

We can realize $w$ as the solution of the IBVP (22)-(25). However, there is value in deriving an alternative form of $F_{j}^{\prime}$. To this end, we introduce a class of test functions $\psi_{j}(t, x), j=1, \ldots, J$, which satisfy

$$
\begin{aligned}
\frac{\partial \psi_{j}}{\partial t} & +\triangle_{x} \psi_{j}=0 \quad x \in \Omega, 0<t<T ; \\
\frac{\partial \psi_{j}}{\partial \eta} & =\rho_{j}, \quad x \in \Gamma, 0<t<T ; \\
\frac{\partial \psi_{j}}{\partial \eta} & =0, \quad x \in S_{0}, 0<t<T ; \\
\psi_{j}(T, x) & =0, \quad x \in \Omega .
\end{aligned}
$$


(Note that the problem is well-posed, since we specify the final condition in the backward heat equation.) The proof of the following key result is rather technical and computational, and is given in an appendix.

Theorem 4.2 For $j=1, \ldots, J$, the following holds:

$$
F_{j}^{\prime}\left(S_{0}\right) \alpha=\left.\int_{\hat{\Omega}_{T}} \alpha(\hat{x})\left(\nabla \psi_{j} \cdot \nabla u+\psi_{j} \frac{\partial u}{\partial t}\right)\right|_{S_{0}} d \hat{x} d t .
$$

We also note that Theorem 4.2 can be extended to the case in which $\Omega$ is unbounded if, e.g., $\tilde{\Omega}=\mathbb{R}^{n-1}$, provided that $\Gamma$ and $S_{0}$ can still be described as the graphs of appropriately smooth functions, and the class of admissible back surfaces is has support in a compact set.

Theorem 4.2 allows us to give a rather short proof that the linearization of the map $\left.\alpha \rightarrow u_{S_{0}+\alpha}\right|_{\Gamma}$ given as $\left.\alpha \rightarrow w\right|_{\Gamma}$, where $w$ satisfies (22)-(25) is injective (so that in principle, one can recover a perturbation $\alpha$ in $S_{0}$ from data on $\Gamma$ ), at least if we have data for all time.

Theorem 4.3 Let $\alpha_{1}$ and $\alpha_{2}$ be admissible perturbations in $S_{0}$ and $w_{1}, w_{2}$ the corresponding solutions to (22)-(25). If $w_{1}(t, x) \equiv w_{2}(t, x)$ for $x \in \Gamma$ and all $t>0$ then $\alpha_{1}=\alpha_{2}$ (provided the input flux $g$ is not identically zero).

Proof. We need only show that if $w$ satisfying (22)-(25) is identically zero on $(0, \infty) \times \Gamma$ then $\alpha \equiv 0$. To this end, consider taking, for any given fixed $T>0$, a test function $\psi$ satisfying (33)-(36) with $\rho_{j}(t, x)=u(T-t, x)$ for $x \in \Gamma$, so that we have $\psi(t, x)=u(T-t, x)$ satisfies (22)-(25). If $F$ is the corresponding functional defined by equation (31) then we have $F^{\prime}\left(S_{0}\right) \alpha=0$ for all $\alpha$, since (from equation (32)) we have $w=0$ on $\Gamma_{T}$. As a result we have

$$
\int_{\hat{\Omega}_{T}} \alpha(\hat{x})\left(\nabla u(T-t, x) \cdot \nabla u(t, x)+u(T-t, x) \frac{\partial u}{\partial t}(t, x)\right) d \hat{x} d t=0
$$

for all $T>0$. Laplace transform both sides of equation (38) with respect to $T$ (certainly permissible since $u$ will not grow rapidly for any reasonable input flux $g$, e.g., any bounded input flux); let $U(s, x)$ denote the transform. We obtain

$$
\int_{\hat{\Omega}} \alpha(\hat{x})\left(|\nabla U(s, x)|^{2}+s U^{2}(s, x)\right) d \hat{x}=0
$$

for all $s>0$, from which we conclude that $\alpha \equiv 0$ (since $U$ is certainly not identically zero for any non-zero input flux $g$ ).

The basis of the reconstruction algorithm is a rather-straightforward implementation of Newton's method for the functional $F: \mathcal{P}_{\gamma} \rightarrow \mathbb{R}^{J}$. At each iteration we compute $F$ (by numerically solving the forward problem (1)-(4) with the current back surface estimate $S$ ). 
We then compute the functions $\psi_{j}$ by numerically solving (33)-(36) (though this can be done rapidly by re-using the computations done to solve for $u$ ). Finally, we compute the Jacobian of $F$ using equation (37) and update the estimate of the back surface $S$. More details and examples are given in the next section.

\section{$5 \quad$ Numerical Examples}

We illustrate the above framework with some computational examples on a two-dimensional domain. We will use cartesian coordinates $x=\left(x_{1}, x_{2}\right)$ and assume, using the notation of Section 2 , that the uncorroded reference domain $\Omega$ is a "medicine-capsule" shaped region defined by the rectangular region $-10 \leq x_{1} \leq 10,-1<x_{2}<0$, together with a half disk of radius $1 / 2$ at each end, centered at $(-10,-1 / 2)$ and $(10,-1 / 2)$. This domain satisfies the hypotheses of the previously stated theorems. The accessible front surface $\Gamma$ will consist of the top half of the region (that portion of $\partial \Omega$ lying above $x_{2}=-1 / 2$ ) and $S_{0}$ the bottom half. In the case of corrosion we assume that $S_{0}$ is of the form $x_{2}=-1+\sigma\left(x_{1}\right)$ for some function $\sigma$ supported in the interval $[-5,5]$. Let $u_{S_{0}}(t, x)$ denote the solution to equations (1)-(4).

Without going into great detail, we remark that in the implementation of our algorithm, we solve the forward problem by converting (1)-(4) into a boundary integral equation on $\partial \Omega \times(0, T)$, which yields

$$
\begin{aligned}
\frac{1}{2} u(T, x) & +\int_{0}^{T} \int_{\partial \Omega} \frac{\partial G}{\partial n_{y}}(T-t, y-x) u(t, y) d s_{y} d t \\
& =\int_{\Omega} G(T, y-x) u_{0}(x) d y+\int_{0}^{T} \int_{\partial \Omega} G(T-t, t-x) g(t, y) d s_{y} d t
\end{aligned}
$$

where $y=\left(y_{1}, y_{2}\right)$ and $G(t, y-x)=\frac{1}{4 \pi t} e^{-\frac{|x-y|^{2}}{4 t}}$ is the standard Green's function for the heat operator in $\mathbb{R}^{2}$. We then solve the integral equation numerically by representing $u(t, x)=\sum_{k=1}^{M} c_{k}(t) \phi_{k}(x)$ for suitable basis functions $\phi_{k}(x)$ defined on $\partial \Omega$. Inserting this expansion into (39) and collocating at points $x=x^{j}$ yields a set of integral equations for the $c_{k}(t)$ which we solve with a simple marching scheme based on the trapezoidal rule. We can then recover the solution $u(t, x)$ for $x \in \Gamma$. Based on comparison to closed-form solutions, our numerical solver is accurate to about 4 significant figures.

\section{Newton's Method Details}

Define a function $F(S)=\left(F_{1}(S), \ldots, F_{J}(S)\right)$ where $F_{j}(S)$ is defined by equation (31) and in which $d(x, t)$ denotes the measured front surface data corresponding to the unknown surface $S_{0}$, with $\rho_{j}(x, t)$ chosen weight functions (to be specified shortly). As remarked previously, our goal is to find a "root" for the function $F$ in the set of admissible surfaces $\mathcal{P}$, by using 
Newton's method. The functions $\rho_{j}$ should (ideally) be chosen to extract as much useful information as possible from the data $d$ (and yet can also be used judiciously to regularize the inversion).

Let $\alpha$ be an admissible perturbation of $S$. From Theorem 4.2 we have, up to linearization, $F_{j}(S+\alpha)-F_{j}(S)=F_{j}^{\prime}(S) \alpha$ where $F_{j}^{\prime}(S) \alpha$ is given by equation (37) and where $\psi_{j}$ satisfies equations (33)-(36). Then

$$
F^{\prime}(S)(\alpha)=\left(F_{1}^{\prime}(S) \alpha, \ldots, F_{J}^{\prime}(S) \alpha\right)
$$

is the linearization of $F$ at $S$.

Newton's method proceeds in the usual manner: We begin with an initial guess $S=S^{0}$, then set $S^{k+1}=S^{k}+\alpha^{k}$ for $k=0,1,2, \ldots$, where $\alpha^{k}$ satisfies $F^{\prime}\left(S^{k}\right) \alpha^{k}=-F\left(S^{k}\right)$, and iterate until some termination condition is met. Of course the equation $F^{\prime}\left(S^{k}\right) \alpha=-F\left(S^{k}\right)$ for the update function $\alpha^{k}$ is undetermined. To remedy this, we seek that (unique) leastsquares $L^{2}$ solution $\alpha$ of minimum norm (an idea that has been used before, in conjunction with the test function approach, in [4] and [9]). A standard Lagrange multiplier computation shows that this minimizer is of the form

$$
\alpha\left(x_{1}\right)=\sum_{k=1}^{J} \lambda_{k} \phi_{k}\left(x_{1}\right)
$$

where

$$
\phi_{k}(x)=\int_{0}^{T}\left(\nabla_{x} \psi_{k}(t, x, S(x)) \cdot \nabla_{x} u_{S}(t, x, S(x))+\psi_{k}(t, x, S(x)) \frac{\partial u_{S}}{\partial t}(t, x, S(x))\right) d t
$$

Note that the functions $\phi_{k}$ as defined are in fact smooth. The coefficients $\lambda_{k}$ can be obtained by using $\alpha$ as defined by equation (40) in the equation $F^{\prime}\left(S^{j}\right) \alpha^{j}=-F\left(S^{j}\right)$, which then distills down to a system $\mathbf{M} \Lambda=\mathbf{b}$ of $J$ linear equations in $J$ unknowns with

$$
M_{j k}=\int_{\Gamma} \phi_{j}(x) \phi_{k}(x) d x
$$

$\mathbf{b}=-F\left(S^{j}\right)$, and $\Lambda=\left(\lambda_{1}, \ldots, \lambda_{J}\right)^{T}$. The matrix $\mathbf{M}$ is positive semi-definite. To see this simply note that for any vector $\mathbf{v}$ we have

$$
\mathbf{v}^{T} \mathbf{M} \mathbf{v}=\int_{\Gamma} \sum_{j, k=1}^{J} v_{j} v_{k} \phi_{j}(x) \phi_{k}(x) d x=\int_{\Gamma}\left(\sum_{j=1}^{J} v_{j} \phi_{j}(x)\right)^{2} d x \geq 0 .
$$

It's also clear that equality will be obtained in equation (42) (so $\mathbf{M}$ will be singular) precisely when $\sum_{j=1}^{J} v_{j} \phi_{j}(x) \equiv 0$ on $\Gamma$.

The minimum-norm/least-squares solution to $F(S)=0$ can be obtained by factoring $\mathbf{M}$ as $\mathbf{M}=\mathbf{P D P}^{T}$ with $\mathbf{P}$ orthogonal and $\mathbf{D}$ diagonal with non-negative entries $D_{k}, k=1$ to $J$ (possible since $\mathbf{M}$ is symmetric and positive semi-definite) then setting $\lambda_{k}=\mathbf{P y}$ where $\mathbf{y}$ is the $n$-vector with components $y_{k}$ defined by $y_{k}=\left(\mathbf{P}^{T} \mathbf{b}\right)_{k} / D_{k}$ for $D_{k}>0, y_{k}=0$ for $D_{k}=0$. 


\section{Regularization}

In fact, we can further regularize the reconstruction in the presence of noise by taking $\Lambda=\mathbf{P y}$ but with $\mathbf{y}$ defined by

$$
y_{k}= \begin{cases}\frac{\left(\mathbf{P}^{T} \mathbf{b}\right)_{k}}{D_{k}}, & D_{k}>\delta \\ 0, & D_{k} \leq \delta\end{cases}
$$

for some $\delta>0$ chosen intelligently in accordance with the noise level of the data and geometry of the sample. To illustrate, let us consider the case described above (the "medicinecapsule" uncorroded reference domain previously described), and suppose the data $d(x, t)$ contains additive noise $r(x, t)$ with $|r(x, t)| \leq R$ for all $x, t$. The vector e with components

$$
e_{k}=\int_{\Gamma \times(0, T)} r(x, t) \rho_{k}(x, t) d x d t
$$

is the error induced in $\mathbf{b}=-F\left(S^{j}\right)$. Let $\alpha_{r}=\sum_{k} \epsilon_{k} \phi_{k}(x)$ be the error induced in our estimate of $\alpha$ by the noise, where the vector $\epsilon=\left(\epsilon_{1}, \ldots, \epsilon_{J}\right)$ is computed from e using equation (43), so $\epsilon=P \mathbf{y}$ where $y_{k}=\left(\mathbf{P}^{T} \mathbf{e}\right)_{k} / D_{k}$ for $D_{k}>\delta, y_{k}=0$ for $D_{k} \leq \delta$.

We have $\left|e_{k}\right| \leq R \int_{\Gamma \times(0, T)}\left|\rho_{k}(x, t)\right| d x d t$ and so

$$
\|\mathbf{e}\|_{2} \leq R \sqrt{\sum_{k} \int_{\Gamma \times(0, T)}\left|\rho_{k}(x, t)\right| d x d t}
$$

where $\|\mathbf{e}\|_{2}$ is the usual 2-norm of the vector $\mathbf{e}$. From equation (43) and $\mathbf{M}=\mathbf{P D P}^{T}$ with $\mathbf{P}$ orthogonal we then have

$$
\|\epsilon\|_{2}=\left\|\mathbf{P}^{T} \epsilon\right\|_{2} \leq \frac{\left\|\mathbf{P}^{T} \mathbf{e}\right\|_{2}}{\delta}=\frac{\|\mathbf{e}\|_{2}}{\delta} .
$$

From $\alpha_{r}=\sum_{k} \epsilon_{k} \phi_{k}(x)$ also have

$$
\begin{aligned}
\left\|\alpha_{r}\right\|_{2} & =\left|\sum_{k} \epsilon_{k} \phi\right|_{2} \\
& \leq \sum_{k}\left|\epsilon_{k}\right|\left\|\phi_{k}\right\|_{2} \\
& \leq\|\epsilon\|_{2} \sqrt{\sum_{k}\left\|\phi_{k}\right\|_{2}^{2}}
\end{aligned}
$$

where $\left\|\phi_{k}\right\|_{2}$ is the usual two norm of $\phi_{k}$ as a function on $\Gamma$. Combining (45)-(47) yields

$$
\left\|\alpha_{r}\right\|_{2} \leq \frac{R}{\delta}\left(\sum_{k}\left\|\phi_{k}\right\|_{2}^{2}\right)^{1 / 2}\left(\sum_{k} \int_{\Gamma \times(0, T)}\left|\rho_{k}(x, t)\right| d x d t\right)^{1 / 2}
$$


Now let us place a bound, $\left\|\alpha_{R}\right\|_{2} \leq B$, on the size of the allowable error $\alpha_{r}$ in the Newton step due to the noise. From the inequality (48), such a bound is obtained if we require $\delta$ to satisfy

$$
\delta \geq \frac{R}{B}\left(\sum_{k}\left\|\phi_{k}\right\|_{2}^{2}\right)^{1 / 2}\left(\sum_{k} \int_{\Gamma \times(0, T)}\left|\rho_{k}(x, t)\right| d x d t\right)^{1 / 2}
$$

Note that all quantities on the right in (49) are known (or estimated) a priori, or computed in the course of the Newton iteration.

In particular, in the "noise-free" examples which follow we use $R=10^{-4}$ (roughly the accuracy of our forward solver, based on comparison to closed form solutions) and $B=0.3$ (our $\sigma$ function is supported within the interval $[-5,5]$ and we limit the stepsize due to noise to uniform magnitude 0.03 over that interval). We use 10 test functions $\rho_{k}$, translates of

simple Gaussian functions (specified below), yielding $\left(\sum_{k} \int_{\Gamma \times(0, T)}\left|\rho_{k}(x, t)\right| d x d t\right)^{1 / 2} \approx 5.1$. The examples all converge in at most 5 iterations, and we compute, as part of the Newton iterations, $\left(\sum_{k}\left\|\phi_{k}\right\|_{2}^{2}\right)^{1 / 2} \approx 0.21$ for the initial iteration (but this quantity ranges up to 0.61 at the final iteration). We then obtain from equation (49) the bound $\delta=2.9 \times 10^{-4}$ for the first Newton iteration, up to $\delta=7.8 \times 10^{-4}$ for the final iteration. The actual values of the $D_{j}$ on the first iteration range from $9.5 \times 10^{-3}$ down to $1.7 \times 10^{-5}$, and 3 of 10 fall below $\delta$. See Figure 1 for plots of the decay rate of the $D_{j}$ for varying numbers of test functions.

We make one additional modification to Newton's method, to improve the global convergence: when solving for the Newton update $\Lambda=\mathbf{P y}$ where $\mathbf{y}$ is given by equation (43), we require that $\|\mathbf{y}\|_{2} \leq d$ for some constant $d$, i.e., we bound the stepsize. A straightforward Lagrange-multiplier argument shows that such a solution is unique and given either by the procedure already outlined (so $\|\Lambda\|<d$ and the stepsize constraint is not active) or $\Lambda=\mathbf{P D}\left(\mathbf{D}^{2}+\eta \mathbf{I}\right)^{-1} \mathbf{P}^{T} \mathbf{b}$ for a unique choice of $\eta>0$, if the stepsize constraint is active.

\section{Examples}

The reconstructions below are for surfaces $S_{0}$ defined by $x_{2}=\sigma_{1}\left(x_{1}\right)$ and $x_{2}=\sigma_{2}\left(x_{1}\right)$ with $\sigma_{1}(x)=0.4 h(0.2(x+2))+0.2 h(0.3(x+4))$ and $\sigma_{2}(x)=0.6 h(0.8(x-0.5))+0.5 h(0.9(x+$ $1))+0.2 h(0.3(x+4))$ where $h(x)$ is defined as $h(x)=16 x^{2}(1-x)^{2}$ for $0<x<1$, and $h(x) \equiv 0$ otherwise.

In each case we use time interval $(0,1 / 2)$, with the temperature $u$ measured at points $x_{1}=-4+0.2 k, k=0$ to $k=40$ on the top surface $x_{2}=0$ (the interval $[-4,4]$ on the $x_{1}$ axis) at each of times $t=0.05 j, j=1$ to $j=10$. There is considerable leeway (and analysis which could be done) in picking the functions $\rho_{j}$. Since we seek to detect "localized" corrosion (confined to the interval $x_{1} \in[-5,5]$ on the back surface) it seems reasonable to use test functions with relatively compact support contained in this interval on the front surface, where we expect most of the information contained in the data to lie. As such, we 
take test functions

$$
\rho_{j}(x)=e^{-c\left(x-a_{j}\right)^{2}}
$$

with $c=55 / 4, a_{j}=-4+8 j / 11$ for $j=1$ to $j=10$, yielding 10 equi-spaced Gaussians along the top surface $\Gamma$; note that the $\rho_{j}$ can depend on time, though ours do not. Indeed, an interesting question for further analysis is that of how the $\rho_{j}$ should vary with timeprevious analysis (see [6]) demonstrates that there is an "optimal time window" in the measured data for the stable recovery of the back surface.

The choice of input flux $g$ can also have a considerable impact on one's ability to see the back surface, and there is also further analysis one could do on the "optimal" input flux. We simply take $g\left(t, x_{1}\right)=\delta(t)$ for $-10<x_{1}<10$, where $\delta(t)$ denotes a Dirac delta function in time; this is a common model for a "flash lamp" input flux. Although this may appear to violate the conditions involved earlier in the analysis of the inverse problem, we can put things into the earlier analytical framework by considering $u_{S}(t, x)-u_{0}(t, x)$, where $u_{0}$ denotes the solution to the heat equation on a half space with Neumann data $\delta(t)$.

We should note that although we use the same general technique to generate the boundary data and implement Newton's method, we attempt to mitigate any "inverse crime" by choosing different basis functions and discretization parameters for the solver.

Since the underlying inverse problem here is quite ill-posed, one expects that any scheme such as this - in which test functions are used to match moments of the measured and computed data - will yield increasingly ill-posed systems of equations as the number of test functions increases. In particular, we expect that if one uses a large number of test functions then the singular values $D_{k}$ of the matrix $\mathbf{M}$ will decay very rapidly. A steadystate version of this problem was examined in [4]. To illustrate the nature of the ill-posedness in the present setting, we plot below the singular values of the matrix $\mathbf{M}$ obtained using each of 10,20, and 30 Gaussian test functions as defined by equation (50), equi-spaced on the interval $[-4,4]$, but with widths scaled according to the number of test functions (so $c=55 / 4,55$, and $495 / 4$ for 10,20, and 30 test functions, respectively). The resulting singular values, plotted on a logarithmic scale, look like 


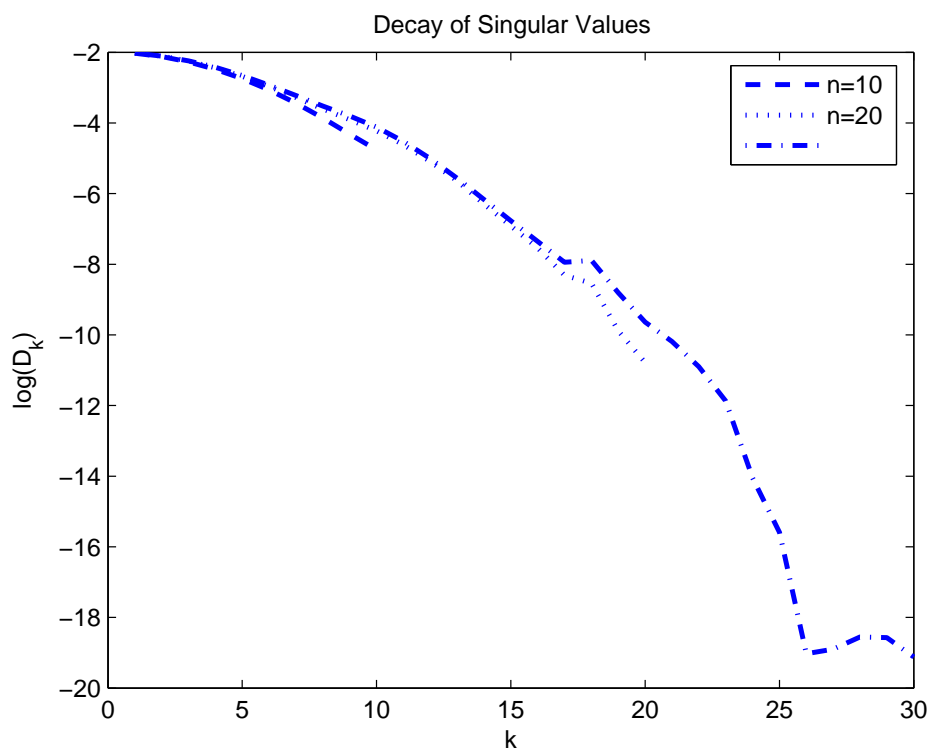

Figure 1: Decay of singular values for $\mathbf{M}$.

Given that our cutoff for the $D_{k}$ typically range from $10^{-4}$ to $10^{-3}$, it is clear that regardless of the number and spacing of test functions used, there is a very limited amount of usable information in the data.

The two graphs below show the reconstructions of $\sigma_{1}$ and $\sigma_{2}$ based on noise-free (up to numerical solver accuracy) data, but with regularization based on noise level $R=10^{-4}$. The solid curves are the actual functions and the dashed curves the reconstructions.
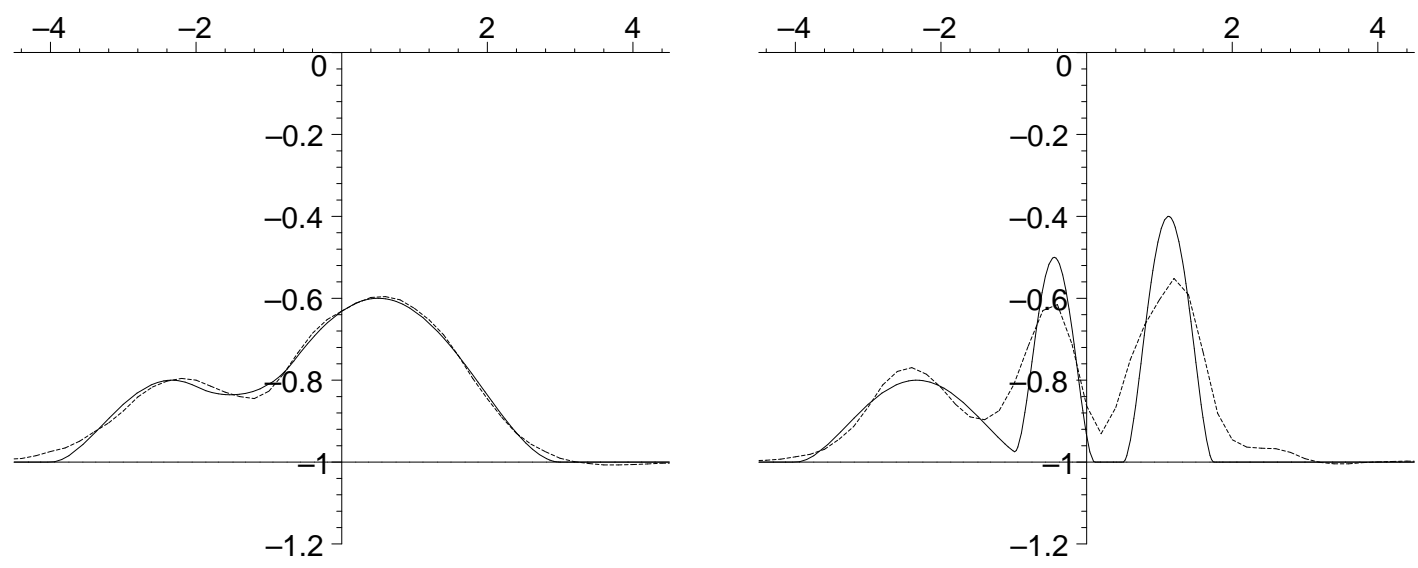

Figure 2: Reconstruction for $\sigma_{1}(x)$ (left) and $\sigma_{2}(x)$ (right), noise-free. 
In each case the Newton method was started with $\sigma \equiv 0$ as an initial guess and converged in just 3 iterations, reducing the residual error $\sum_{k} F_{k}^{2}(S)$ by a factor of 20 to 60 . Even the rather large amplitude and oscillatory back surface described by function $\sigma_{2}$ is reasonably well recovered with this level of noise.

In the second pair of reconstructions below we use $\sigma_{1}$ and $\sigma_{2}$ as above, but add noise to the data $d(t, x)$. At each data point (time/space) we added independent Gaussian random noise, standard deviation 0.05 . For reference purposes, the temperature $u$ lies in the range $u=1$ to $u=2.5$ over the course of the simulation. The perturbation in the temperature, as compared to the uncorroded domain with back surface $\sigma=0$, is about 0.5 , so the noise is approximately 10 percent of the signal magnitude.

Newton's method again converges in three to four iterations, reducing the residual by a factor of 5 to 12 . The value of $\delta$ in equation (49) ranges around 0.01 , and typically eliminates about half of the $y_{k}$ in equation (43).
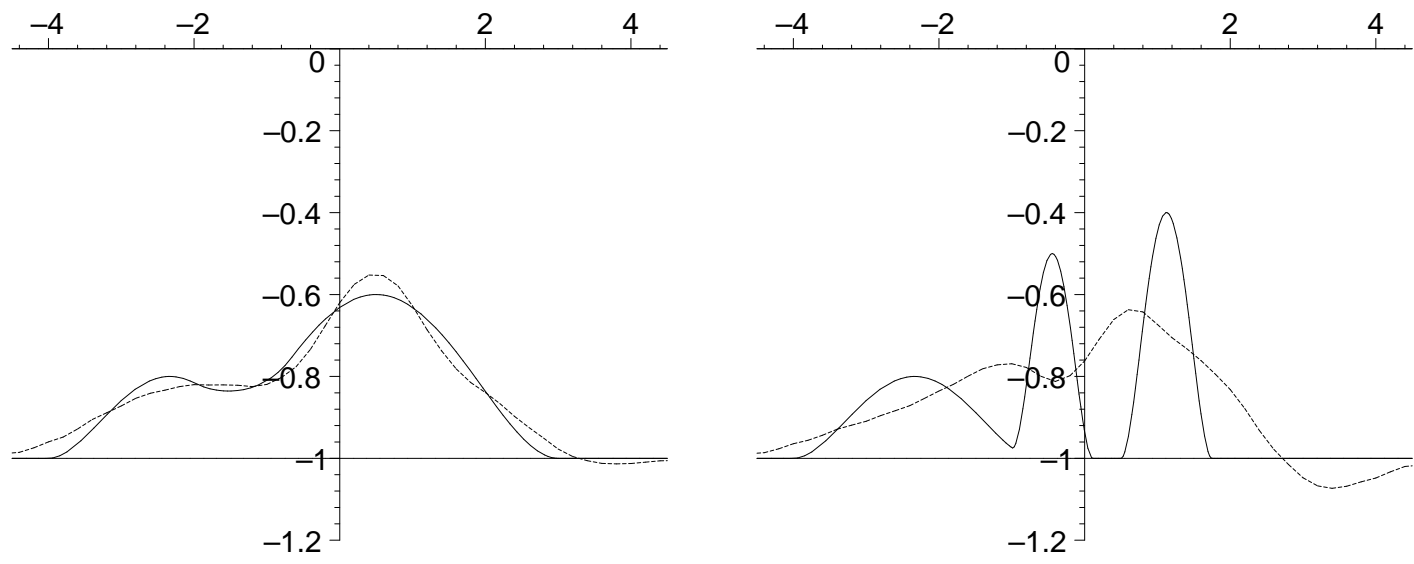

Figure 3: Reconstruction for $\sigma_{1}(x)$ (left) and $\sigma_{2}(x)$ (right), noisy.

As one would expect, the surface $\sigma_{2}$ is not as well recovered, but rather somewhat blurred, though the location and magnitude of the corrosion is reasonably estimated.

\section{Conclusions}

We have presented an algorithm for determining an unknown boundary portion of a domain from a single Cauchy data pair. This algorithm offers the advantage that it is applicable to a wide class of domains in $\mathbb{R}^{n}$. We have also given an explicit expression for the Jacobian of the relevant vector-valued functional. The effectiveness of this algorithm has been demonstrated on test examples. 
The methods used in the present work can be extended in a straightforward way to apply to more general operators and boundary conditions. For example, the Laplacian of equation (1) can be replaced with a more general elliptic operator of the form

$$
\nabla \cdot(a(\hat{x}) \nabla u)
$$

Also, the Neumann boundary conditions (2) and (3) can be generalized to the Robin conditions

$$
\frac{\partial u}{\partial \eta}+r u=g(t, x)
$$

for (2), and the obvious analogue for (3).

This work raises important and interesting issues relating to the optimal design of such thermal imaging experiments. From physical principles as well as numerical experiments, it is clear that the specific choices of such ingredients as the time interval $(0, T)$, the input flux $g(t, x)$ of equation (2), and even the test functions $\rho_{j}$ used in the definition (31) of the functionals $F_{j}$, will bear significantly on the important questions of stability and resolution. These issues are presently under investigation.

\section{References}

[1] Banks, H.T., and Kojima, F., Boundary shape identification problems in twodimensional domains related to thermal testing of materials, Quart. Appl. Math. 47 (1989), 273-293.

[2] Banks, H.T., Kojima, F., and Winfree, W.P., Boundary estimation problems arising in thermal tomography, Inverse Problems 6 (1990), 897-922.

[3] Bryan, K. and Caudill, L., Stability and resolution in thermal imaging, Proceedings of the 1995 ASME Design Engineering Technical Conference, Boston, 1995, 1023-1032.

[4] Bryan, K. and Caudill, L., An inverse problem in thermal imaging, SIAM J. Appl. Math. 59 (1996), 715-735.

[5] Bryan, K. and Caudill, L., Uniqueness for a boundary identification problem in thermal imaging, Electron. J. Differential Equations C-1 (1997), 23-39.

[6] Bryan, K. and Caudill, L., Stability and reconstruction for an inverse problem for the heat equation, Inverse Problems 14 (1998), 1429-1453.

[7] Canuto, B., Rosset, E., and Vessella, S., Quantitative estimates of unique continuation for parabolic equations and inverse initial-boundary value problems with unknown boundaries, it Trans. Amer. Math. Soc. 354 (2002), 491-535. 
[8] Chapko, R., Kress, R., and Yoon, J-R., An inverse boundary value problem for the heat equation: The Neumann condition, Inverse Problems 15 (1999), 1033-1049.

[9] Dobson, D., and Santosa, F., Stability and resolution analysis of an inverse problem in electrical impedance tomography - dependence on input current patterns, SIAM J. Appl. Math., 54, (1994), 1542-1560.

[10] Ladyzenskaja, O., Solonnikov, V., and Ural'ceva, N., Linear and Quasi-linear Equations of Parabolic Type, American Mathematical Society, 1968.

[11] Vessella, S., Stability estimates in an inverse problem for a three-dimensional heat equation, SIAM J. Math. Anal. 28 (1997), 1354-1370.

\section{Appendix: Proof of Theorem 4.2}

A bit of notation: We will use the following shorthand for certain important vectors:

$$
\begin{aligned}
& \hat{\gamma}=\left(\gamma_{x_{1}}, \ldots, \gamma_{x_{n-1}},-1\right)^{T} \\
& \hat{\sigma}=\left(\sigma_{x_{1}}, \ldots, \sigma_{x_{n-1}},-1\right)^{T} \\
& \hat{\alpha}=\left(\alpha_{x_{1}}, \ldots, \alpha_{x_{n-1}}, 0\right)^{T} .
\end{aligned}
$$

Then, the unit normal vectors $\eta$ on the various surfaces can be written

$$
\begin{array}{ccc}
\eta=\frac{\hat{\gamma}}{\|\hat{\gamma}\|} & \text { on } & \Gamma, \\
\eta=\frac{\hat{\sigma}}{\|\hat{\sigma}\|} & \text { on } & S_{0}, \\
\eta=\frac{\hat{\sigma}+\epsilon \hat{\alpha}}{\|\hat{\sigma}+\epsilon \hat{\alpha}\|} & \text { on } & S_{0}+\epsilon S .
\end{array}
$$

The vector $\vec{\psi}$, which will prove important in the sequel, is defined as follows:

$$
\vec{\psi}= \begin{cases}\rho_{\epsilon}\|\hat{\gamma}\| \eta, & \text { on } \Gamma ; \\ -\nabla\left(\rho_{\epsilon}\left(x_{n}-\gamma(\hat{x})\right)\right), & \text { on } \Omega ; \\ -\hat{\alpha}+\rho_{\epsilon}\|\hat{\sigma}\| \eta, & \text { on } S_{0}\end{cases}
$$

A few preliminary calculations now will streamline the derivation to come. These results can be easily verified by direct calculation. ( $\Psi_{\epsilon}$ is the Gateaux derivative of $\Psi$.)

$$
\nu_{\epsilon}=\Psi_{\epsilon} \eta+\eta_{\epsilon},
$$


where

$$
\begin{gathered}
\eta_{\epsilon}= \begin{cases}0 & \text { on } \Gamma, \\
\frac{\hat{\alpha}}{\|\hat{\sigma}\|}-\left(\frac{\hat{\alpha} \cdot \hat{\sigma}}{\|\hat{\sigma}\|^{2}}\right) \eta & \text { on } S_{0} .\end{cases} \\
\eta_{n}= \begin{cases}-\frac{1}{\|\hat{\gamma}\|} & \text { on } \Gamma, \\
-\frac{1}{\|\hat{\sigma}\|} & \text { on } S_{0} .\end{cases} \\
\rho_{\epsilon}\left(x_{n}-\gamma(\hat{x})\right)= \begin{cases}0 & \text { on } \Gamma, \\
\alpha(\hat{x}) & \text { on } S_{0} .\end{cases}
\end{gathered}
$$

Finally, if $\hat{y}$ and $\hat{z}$ are vectors in $\mathbb{R}^{n}$, then the following hold:

$$
\begin{aligned}
& \hat{y} \cdot\left(\Psi_{\epsilon} \hat{z}\right)=y_{n}(\vec{\psi} \cdot \hat{z}) \\
& \hat{y} \cdot\left(\kappa_{\epsilon} \hat{z}\right)=\rho_{\epsilon}(\hat{y} \cdot \hat{z})+\vec{\psi} \cdot\left(y_{n} \hat{z}+z_{n} \hat{y}\right)
\end{aligned}
$$

Proof of Theorem 4.2: Multiply the left-hand side of (22) by $\psi$, integrate over $\Omega_{T}$, and use Green's identities, as well as equations (25) and (35) to obtain:

$\int_{\partial \Omega_{T}} w \frac{\partial \psi}{\partial \eta} d s d t=\int_{\partial \Omega_{T}} \psi \frac{\partial w}{\partial \eta} d s d t+\int_{\partial \Omega_{T}} \psi \nabla u \cdot\left(\kappa_{\epsilon} \eta\right) d s d t-\int_{\Omega_{T}} \nabla \psi \cdot\left(\kappa_{\epsilon} \nabla u\right) d x d t-\int_{\Omega_{T}} \rho_{\epsilon} \psi \frac{\partial u}{\partial t} d x d t$.

Note that, by virtue of (34), the LHS integral is zero except possibly on $\Gamma$. Using (23) and (24) in the first RHS integral, and (58) in the second and third RHS integrals yields

$$
\begin{aligned}
\int_{\Gamma_{T}} w \frac{\partial \psi}{\partial \eta} d s d t= & -\int_{\partial \Omega_{T}} \psi \frac{\partial u}{\partial \nu_{\epsilon}} d s d t+\int_{\partial \Omega_{T}} \rho_{\epsilon} \psi \frac{\partial u}{\partial \eta} d s d t+\int_{\partial \Omega_{T}} \psi \frac{\partial u}{\partial x_{n}}(\vec{\psi} \cdot \eta) d s d t \\
& +\int_{\partial \Omega_{T}} \psi \eta_{n} \vec{\psi} \cdot \nabla u d s d t-\int_{\Omega_{T}} \rho_{\epsilon}(\nabla \psi \cdot \nabla u) d x d t \\
& -\int_{\Omega_{T}} \vec{\psi} \cdot\left(\frac{\partial \psi}{\partial x_{n}} \nabla u+\frac{\partial u}{\partial x_{n}} \nabla \psi\right) d x d t-\int_{\Omega_{T}} \rho_{\epsilon} \psi \frac{\partial u}{\partial t} d x d t \\
\equiv & C_{11}+C_{12}+C_{13}+C_{14}+C_{15}+C_{16}+C_{17} .
\end{aligned}
$$

Using (53) in $C_{11},(55)$ in $C_{14}$, and (52) in $C_{14}$ and $C_{16}$ in equation (59) yields 


$$
\begin{aligned}
\int_{\Gamma_{T}} w \frac{\partial \psi}{\partial \eta} d s d t= & -\int_{\partial \Omega_{T}} \psi \nabla u \cdot\left(\Psi_{\epsilon} \eta\right) d s d t-\int_{\partial \Omega_{T}} \psi \frac{\partial u}{\partial \eta_{\epsilon}} d s d t+\int_{\partial \Omega_{T}} \rho_{\epsilon} \psi \frac{\partial u}{\partial \eta} d s d t \\
& +\int_{\partial \Omega_{T}} \psi \frac{\partial u}{\partial x_{n}}(\vec{\psi} \cdot \eta) d s d t-\int_{\Gamma_{T}} \frac{1}{\|\hat{\gamma}\|} \rho_{\epsilon}\|\hat{\gamma}\| \psi \frac{\partial u}{\partial \eta} d s d t+\int_{S_{T}} \frac{1}{\|\hat{\sigma}\|} \psi(\nabla u \cdot \hat{\alpha}) d s d t \\
& -\int_{S_{T}} \frac{1}{\|\hat{\sigma}\|} \rho_{\epsilon}\|\hat{\sigma}\| \psi \frac{\partial u}{\partial \eta} d s d t \\
& -\int_{\Omega_{T}} \rho_{\epsilon}(\nabla \psi \cdot \nabla u) d x d t+\int_{\Omega_{T}} \nabla\left(\rho_{\epsilon}\left(x_{n}-\gamma(\hat{x})\right)\right) \cdot\left(\frac{\partial \psi}{\partial x_{n}} \nabla u+\frac{\partial u}{\partial x_{n}} \nabla \psi\right) d x d t \\
& -\int_{\Omega_{T}} \rho_{\epsilon} \psi \frac{\partial u}{\partial t} d x d t \\
\equiv & C_{21}+C_{22}+C_{23}+C_{24}+C_{25}+C_{26}+C_{27}+C_{28}+C_{29}+C_{20} .
\end{aligned}
$$

Note that $C_{23}, C_{25}$, and $C_{27}$ cancel each other, and that (54) implies that $C_{22}$ can be taken over $S_{0}$, rather than all of $\partial \Omega$. Now, apply (57) to $C_{21}$ and integrate by parts with respect to $x$ in $C_{29}$ in equation (60) to obtain

$$
\begin{aligned}
\int_{\Gamma_{T}} w \frac{\partial \psi}{\partial \eta} d s d t= & -\int_{\partial \Omega_{T}} \psi \frac{\partial u}{\partial x_{n}}(\vec{\psi} \cdot \eta) d s d t-\int_{S_{T}} \psi \frac{\partial u}{\partial \eta_{\epsilon}} d s d t+\int_{\partial \Omega_{T}} \psi \frac{\partial u}{\partial x_{n}}(\vec{\psi} \cdot \eta) d s d t \\
& +\int_{S_{T}} \frac{1}{\|\hat{\sigma}\|} \psi(\nabla u \cdot \hat{\alpha}) d s d t-\int_{\Omega_{T}} \rho_{\epsilon}(\nabla \psi \cdot \nabla u) d x d t \\
& +\int_{\partial \Omega_{T}} \rho_{\epsilon}\left(x_{n}-\gamma\right)\left(\frac{\partial \psi}{\partial x_{n}} \frac{\partial u}{\partial \eta}+\frac{\partial u}{\partial x_{n}} \frac{\partial \psi}{\partial \eta}\right) d s d t \\
& -\int_{\Omega_{T}} \rho_{\epsilon}\left(x_{n}-\gamma\right) \nabla \cdot\left(\frac{\partial \psi}{\partial x_{n}} \nabla u+\frac{\partial u}{\partial x_{n}} \nabla \psi\right) d x d t-\int_{\Omega_{T}} \rho_{\epsilon} \psi \frac{\partial u}{\partial t} d x d t \\
\equiv & C_{31}+C_{32}+C_{33}+C_{34}+C_{35}+C_{36}+C_{37}+C_{38} .
\end{aligned}
$$

$C_{31}$ and $C_{33}$ cancel. In $C_{37}$ we will use the identity

$$
\nabla \cdot\left(\frac{\partial \psi}{\partial x_{n}} \nabla u+\frac{\partial u}{\partial x_{n}} \nabla \psi\right)=\frac{\partial}{\partial x_{n}}(\nabla \psi \cdot \nabla u)+\frac{\partial \psi}{\partial x_{n}} \frac{\partial u}{\partial t}-\frac{\partial u}{\partial x_{n}} \frac{\partial \psi}{\partial t},
$$

which in turn utilizes (1) and (33). Noting (56) in $C_{36}$, (61) becomes

$$
\begin{aligned}
\int_{\Gamma_{T}} w \frac{\partial \psi}{\partial \eta} d s d t= & -\int_{S_{T}} \psi \nabla u \cdot \eta_{\epsilon} d s d t+\int_{S_{T}} \frac{1}{\|\hat{\sigma}\|} \psi(\nabla u \cdot \hat{\alpha}) d s d t-\int_{\Omega_{T}} \rho_{\epsilon}(\nabla \psi \cdot \nabla u) d x d t \\
& +\int_{S_{T}} \hat{\alpha}(\hat{x})\left(\frac{\partial \psi}{\partial x_{n}} \frac{\partial u}{\partial \eta}+\frac{\partial u}{\partial x_{n}} \frac{\partial \psi}{\partial \eta}\right) d s d t \\
& -\int_{\Omega_{T}} \rho_{\epsilon}\left(x_{n}-\gamma\right)\left[\frac{\partial}{\partial x_{n}}(\nabla \psi \cdot \nabla u)+\frac{\partial \psi}{\partial x_{n}} \frac{\partial u}{\partial t}\right] d x d t
\end{aligned}
$$




$$
\begin{aligned}
& +\int_{\Omega_{T}} \rho_{\epsilon}\left(x_{n}-\gamma\right) \frac{\partial u}{\partial x_{n}} \frac{\partial \psi}{\partial t} d x d t-\int_{\Omega_{T}} \rho_{\epsilon} \psi \frac{\partial u}{\partial t} d x d t \\
\equiv & C_{41}+C_{42}+C_{43}+C_{44}+C_{45}+C_{46}+C_{47} .
\end{aligned}
$$

Now use (54) in $C_{41}$, integrate by parts with respect to $x_{n}$ in $C_{45}$, and integrate by parts with respect to $t$ (utilizing (4) and (35)) in $C_{46}$, so that (62) becomes

$$
\begin{aligned}
\int_{\Gamma_{T}} w \frac{\partial \psi}{\partial \eta} d s d t= & -\int_{S_{T}} \frac{1}{\|\hat{\sigma}\|} \psi(\nabla u \cdot \hat{\alpha}) d s d t+\int_{S_{T}}\left(\frac{\hat{\alpha} \cdot \hat{\sigma}}{\|\hat{\sigma}\|^{2}}\right) \psi \frac{\partial u}{\partial \eta} d s d t+\int_{S_{T}} \frac{1}{\|\hat{\sigma}\|} \psi(\nabla u \cdot \hat{\alpha}) d s d t \\
& -\int_{\Omega_{T}} \rho_{\epsilon}(\nabla \psi \cdot \nabla u) d x d t+\int_{S_{T}} \alpha(\hat{x})\left(\frac{\partial \psi}{\partial x_{n}} \frac{\partial u}{\partial \eta}+\frac{\partial u}{\partial x_{n}} \frac{\partial \psi}{\partial \eta}\right) d s d t \\
& -\left.\int_{\hat{\Omega}_{T}} \rho_{\epsilon}\left(x_{n}-\gamma\right)\left(\nabla \psi \cdot \nabla u+\psi \frac{\partial u}{\partial t}\right)\right|_{x_{n}=\sigma(\hat{x})} ^{x_{n}=\gamma(\hat{x})} d \hat{x} d t+\int_{\Omega_{T}} \frac{\partial}{\partial x_{n}}\left(\rho_{\epsilon}\left(x_{n}-\gamma\right)\right) \nabla \psi \cdot \nabla u d x d t \\
& +\int_{\Omega_{T}} \frac{\partial}{\partial x_{n}}\left(\rho_{\epsilon}\left(x_{n}-\gamma\right) \frac{\partial u}{\partial t} \psi\right) d x d t-\int_{\Omega_{T}} \rho_{\epsilon}\left(x_{n}-\gamma\right) \frac{\partial^{2} u}{\partial t \partial x_{n}} d x d t-\int_{\Omega_{T}} \rho_{\epsilon} \psi \frac{\partial u}{\partial t} d x d t \\
\equiv & C_{51}+C_{52}+C_{53}+C_{54}+C_{55}+C_{56}+C_{57}+C_{58}+C_{59}+C_{50} .
\end{aligned}
$$

$C_{51}$ and $C_{53}$ cancel. Further, it is easy to verify that

$$
\frac{\partial}{\partial x_{n}}\left(\rho_{\epsilon}\left(x_{n}-\gamma\right)\right)=\rho_{\epsilon}
$$

and

$$
\frac{\partial}{\partial x_{n}}\left(\rho_{\epsilon}\left(x_{n}-\gamma\right) \frac{\partial u}{\partial t} \psi\right)=\rho_{\epsilon} \frac{\partial u}{\partial t}+\rho_{\epsilon}\left(x_{n}-\gamma\right) \frac{\partial^{2} u}{\partial x_{n} \partial t} .
$$

Using these in $C_{57}$ and $C_{58}$, respectively, and using (56) in $C_{56}$ transforms equation (63) into

$$
\begin{aligned}
\int_{\Gamma_{T}} w \frac{\partial \psi}{\partial \eta} d s d t= & \int_{S_{T}}\left(\frac{\hat{\alpha} \cdot \hat{\sigma}}{\|\hat{\sigma}\|^{2}}\right) \psi \frac{\partial u}{\partial \eta} d s d t-\int_{\Omega_{T}} \rho_{\epsilon}(\nabla \psi \cdot \nabla u) d x d t \\
& +\int_{S_{T}} \alpha(\hat{x})\left(\frac{\partial \psi}{\partial x_{n}} \frac{\partial u}{\partial \eta}+\frac{\partial u}{\partial x_{n}} \frac{\partial \psi}{\partial \eta}\right) d s d t+\left.\int_{\hat{\Omega}_{T}} \alpha(\hat{x})\left(\nabla \psi \cdot \nabla u+\psi \frac{\partial u}{\partial t}\right)\right|_{S_{0}} d \hat{x} d t \\
& \int_{\Omega_{T}} \rho_{\epsilon}(\nabla \psi \cdot \nabla u) d x d t+\int_{\Omega_{T}} \rho_{\epsilon} \psi \frac{\partial u}{\partial t} d x d t+\int_{\Omega_{T}} \rho_{\epsilon}\left(x_{n}-\gamma\right) \frac{\partial^{2} u}{\partial x_{n} \partial t} d x d t \\
& -\int_{\Omega_{T}} \rho_{\epsilon}\left(x_{n}-\gamma\right) \frac{\partial^{2} u}{\partial t \partial x_{n}} d x d t-\int_{\Omega_{T}} \rho_{\epsilon} \psi \frac{\partial u}{\partial t} d x d t \\
\equiv & C_{61}+C_{62}+C_{63}+C_{64}+C_{65}+C_{66}+C_{67}+C_{68}+C_{69}
\end{aligned}
$$

$C_{62}$ and $C_{65}$ cancel, as do $C_{66}$ and $C_{69}$, as well as $C_{67}$ and $C_{68}$. Using (3) and (34), we see that $C_{61}=C_{63}=0$, leaving 


$$
\int_{\Gamma_{T}} w \frac{\partial \psi}{\partial \eta} d s d t=\left.\int_{\hat{\Omega}_{T}} \alpha(\hat{x})\left(\nabla \psi \cdot \nabla u+\psi \frac{\partial u}{\partial t}\right)\right|_{S_{0}} d \hat{x} d t .
$$

Thus, Theorem 4.2 is proved. 